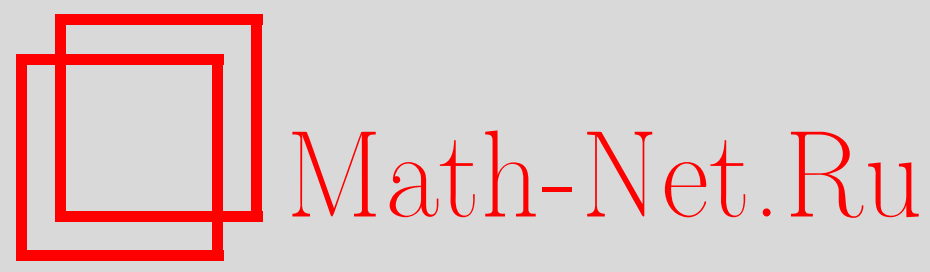

О. Г. Авсянкин, Н. К. Карапетянц, Проекционный метод в теории интегральных операторов с однородными ядрами, Матем. заметки, 2004, том 75, выпуск 2, 163-172

DOI: https://doi.org/10.4213/mzm27

Использование Общероссийского математического портала Math-Net.Ru подразумевает, что вы прочитали и согласны с пользовательским соглашением http://www.mathnet.ru/rus/agreement

Параметры загрузки:

IP : 54.84 .234 .179

26 апреля 2023 г., 09:12:53 


\title{
ПРОЕКЦИОННЫЙ МЕТОД В ТЕОРИИ ИНТЕГРАЛЬНЫХ ОПЕРАТОРОВ С ОДНОРОДНЫМИ ЯДРАМИ
}

\author{
О.Г. Авсянкин, Н. К. Карапетянц
}

В работе исследуется вопрос о применимости проекционного метода к многомерным интегральным операторам с ядрами, однородньми степени $(-n)$ и инвариантньми относительно группы вращений $S O(n)$, в скалярном и матричном случаях.

Библиография: 6 названий.

\section{Введение}

Исследования, посвященные применимости проекционных методов к линейным ограниченным операторам в банаховом пространстве, являются весьма актуальными (см., например, [1]-[3]). В данной работе рассматривается вопрос о применимости проекционных методов к некоторым классам интегральных операторов с однородными ядрами в скалярном и матричном случаях.

Работа состоит из двух разделов. В разделе 1 вводятся необходимые определения и рассматриваются одномерные интегральные операторы с однородными степени $(-1)$ ядрами. Такие операторы являются мультипликативными аналогами операторов свертки, а потому все результаты для них получаются непосредственно из соответствующих результатов для операторов свертки. Основные результаты работы содержатся в разделе 2 , где изучаются многомерные интегральные операторы с однородными степени $(-n)$ ядрами. Для исследования таких операторов применяется специальная методика, основанная на редукции многомерного случая к одномерному. Отметим, что некоторые результаты раздела 2 были анонсированы в [4].

В статье используются следующие обозначения:

$$
\begin{aligned}
& \mathbb{R}^{n}-n \text {-мерное евклидово пространство; } \\
& x=\left(x_{1}, \ldots, x_{n}\right) \in \mathbb{R}^{n} ; \\
& |x|=\sqrt{x_{1}^{2}+\cdots+x_{n}^{2}} ; \\
& x^{\prime}=x /|x| \\
& x \cdot y=x_{1} y_{1}+\cdots+x_{n} y_{n} ; \\
& e_{1}=(1,0, \ldots, 0) ; \\
& S_{n-1}=\left\{x \in \mathbb{R}^{n}:|x|=1\right\} ; \\
& \Omega_{n}=\left\{x \in \mathbb{R}^{n}:|x| \leqslant 1\right\} ; \\
& \dot{\mathbb{R}} \text { - компактификация } \mathbb{R} \text { одной бесконечно удаленной точкой; }
\end{aligned}
$$

Работа поддержана Российским фондом фундаментальных исследований. 
$\mathbb{Z}_{+}-$множество всех целых неотрицательных чисел;

$\mathbb{Z}_{+} \dot{\times} \mathbb{R}-$ компактификация множества $\mathbb{Z}_{+} \times \mathbb{R}$ одной бесконечно удаленной точкой;

$Y_{m \mu}(\sigma)$ - сферические гармоники порядка $m$;

$d_{n}(m)$ - размерность пространства сферических гармоник порядка $m$ :

$$
d_{n}(m)=(n+2 m-2) \frac{(n+m-3) !}{m !(n-2) !}
$$

$L_{p}^{s}(X)$ - пространство $s$-мерных вектор-функций с компонентами из $L_{p}(X)$;

$P_{m}(t)$ - многочлены Лежандра, определяемые равенством

$$
P_{m}(t)= \begin{cases}\cos (m \arccos t), & n=2, \\ \left(C_{m+n-3}^{m}\right)^{-1} C_{m}^{(n-2) / 2}(t), & n \geqslant 3,\end{cases}
$$

где $C_{m}^{(n-2) / 2}(t)$ - многочлены Гегенбауэра.

\section{1. Предварительные сведения и вспомогательные результаты}

1.1. Определение проекционного метода. Пусть $X$ - банахово пространство, $A \in \mathscr{L}(X),\left\{P_{\tau_{1}, \tau_{2}}\right\}, 0<\tau_{1}<1,1<\tau_{2}<\infty,-$ семейство проекторов, действующих в $X$. Будем предполагать, что при $\tau_{1} \rightarrow 0, \tau_{2} \rightarrow \infty$ проекторы $P_{\tau_{1}, \tau_{2}}$ сходятся в сильной операторной топологии к единичному оператору. Рассмотрим уравнение

$$
P_{\tau_{1}, \tau_{2}} A P_{\tau_{1}, \tau_{2}} x=P_{\tau_{1}, \tau_{2}} y
$$

ОПРЕДЕЛЕНИЕ 1. Будем говорить, что к оператору $A$ применим проекиионный метод по системе проекторов $\left(P_{\tau_{1}, \tau_{2}} ; P_{\tau_{1}, \tau_{2}}\right)$ при $\tau_{1} \rightarrow 0$ и $\tau_{2} \rightarrow \infty$, если

1) существуют такие числа $\delta_{1} \in(0,1)$ и $\delta_{2} \in(1, \infty)$, что при всех $\tau_{1}<\delta_{1}$ и $\tau_{2}>\delta_{2}$ для любого $y \in X$ уравнение (1.1) имеет единственное решение $x_{\tau_{1}, \tau_{2}} \in P_{\tau_{1}, \tau_{2}} X$;

$2)$ при $\tau_{1} \rightarrow 0$ и $\tau_{2} \rightarrow \infty$ решение $x_{\tau_{1}, \tau_{2}}$ стремится по норме пространства $X \mathrm{k}$ решению $x \in X$ уравнения $A x=y$.

Класс операторов, к которым применим проекционньй метод по системе проекторов $\left(P_{\tau_{1}, \tau_{2}} ; P_{\tau_{1}, \tau_{2}}\right)$ обозначим через $\Pi\left\{P_{\tau_{1}, \tau_{2}}\right\}$.

Определение 1 эквивалентно тому, что оператор $A$ обратим, при $0<\tau_{1}<\delta_{1}$ и $\delta_{2}<$ $\tau_{2}<\infty$ операторы $P_{\tau_{1}, \tau_{2}} A P_{\tau_{1}, \tau_{2}}$ как операторы, действуюшие в $P_{\tau_{1}, \tau_{2}} X$, обратимы и операторы $\left(P_{\tau_{1}, \tau_{2}} A P_{\tau_{1}, \tau_{2}}\right)^{-1} P_{\tau_{1}, \tau_{2}}$ при $\tau_{1} \rightarrow 0$ и $\tau_{2} \rightarrow \infty$ сильно сходятся к $A^{-1}$.

Очевидно, что все основные теоремы о проекционных методах, доказанные в [1] для случая однопараметрического семейства проекторов, сохраняют справедливость и в нашем случае. В дальнейшем мы будем ссылаться на результаты [1], предполагая, что они переформулированы в соответствии с определением 1.

Необходимость использования двухпараметрического семейства проекторов связана с тем, что рассматриваемые нами интегральные операторы с однородными ядрами имеют две особые точки: “в нуле" и "на бесконечности".

В случае однопараметрического семейства проекторов $\left\{P_{\tau}\right\}, 0<\tau<1$, мы будем также пользоваться определением 1 , не оговаривая это особо. 
1.2. Одномерные интегральные операторы с однородными степени $(-1)$ ядрами. В пространстве $L_{p}(0, \infty)$ (здесь и далее предполагается, что $\left.1 \leqslant p<\infty\right)$ рассмотрим оператор

$$
(\mathscr{H} \varphi)(r)=\int_{0}^{\infty} h(r, \rho) \varphi(\rho) d \rho, \quad r \in(0, \infty),
$$

где функция $h(r, \rho)$

a) однородна степени $(-1)$, т. е.

$$
h(\alpha r, \alpha \rho)=\alpha^{-1} h(r, \rho) \quad \forall \alpha>0 ;
$$

b) удовлетворяет условию $\int_{0}^{\infty}|h(1, \rho)| \rho^{-1 / p} d \rho<\infty$.

Мы будем также рассматривать оператор

$$
(H \varphi)(r)=\int_{0}^{1} h(r, \rho) \varphi(\rho) d \rho, \quad r \in(0,1),
$$

действующий в пространстве $L_{p}(0,1)$.

Теория таких операторов подробно изложена, например, в [5]. Следуя [5], назовем символом операторов $\lambda I-H$ и $\lambda I-\mathscr{H}$ функцию

$$
\sigma(\xi)=\lambda-\int_{0}^{\infty} h(1, \rho) \rho^{-1 / p+i \xi} d \rho, \quad \xi \in \mathbb{R}
$$

Определим в пространстве $L_{p}(0,1)$ проектор $\widetilde{P}_{\tau}, 0<\tau<1$, по формуле

$$
\left(\widetilde{P}_{\tau} \varphi\right)(r)= \begin{cases}\varphi(r), & \tau<r<1 \\ 0, & 0<r<\tau .\end{cases}
$$

ПРЕДЛОЖЕНИЕ 1. Для того чтобы оператор $\lambda I-H \in \Pi\left\{\widetilde{P}_{\tau}\right\}$, необходимо $u$ достаточно, чтобы оператор $\lambda I-H$ был обратим в $L_{p}(0,1)$.

ДокАЗАТЕЛЬСтво. Определим оператор $W_{p}: L_{p}(0,1) \rightarrow L_{p}(0, \infty)$ по формуле

$$
\left(W_{p} \varphi\right)(t)=e^{-t / p} \varphi\left(e^{-t}\right)
$$

Как известно (см. [5]), оператор $B=W_{p} H W_{p}^{-1}$ является оператором Винера-Хопфа, действующим в пространстве $L_{p}(0, \infty)$. Применяя к оператору $B$ теорему 3.1 главы III книги [1] по системе проекторов $\left(W_{p} \widetilde{P}_{\tau} W_{p}^{-1}\right)$, получаем требуемый результат.

Далее, определим в пространстве $L_{p}(0, \infty)$ проектор $\widetilde{P}_{\tau_{1}, \tau_{2}}, 0<\tau_{1}<1,1<\tau_{2}<\infty$, по формуле

$$
\left(\widetilde{P}_{\tau_{1}, \tau_{2}} \varphi\right)(r)= \begin{cases}\varphi(r), & \tau_{1}<r<\tau_{2}, \\ 0, & r<\tau_{1} \text { или } r>\tau_{2} .\end{cases}
$$


ПРЕДЛОЖЕНИЕ 2. Для того чтобы оператор $\lambda I-\mathscr{H} \in \Pi\left\{\widetilde{P}_{\tau_{1}, \tau_{2}}\right\}$, необходимо и достаточно, чтобы выполнялись следующие условия:

1) $\sigma(\xi) \neq 0$ для всех $\xi \in \dot{\mathbb{R}}$

2) ind $\sigma(\xi)=0$.

ДокАЗАТЕЛЬСТВо аналогично доказательству предложения 1.

ЗАмЕчАниЕ 1. Следует отметить, что необходимым и достаточным условием обратимости оператора $\lambda I-\mathscr{H}$ в $L_{p}(0, \infty)$ является только одно условие 1$)$, но для применимости проекционного метода необходимо и достаточно вьполнения двух условий: 1) и 2).

Далее, в пространстве $L_{p}^{s}(0,1)$ рассмотрим оператор $H \equiv H_{s}$ вида $(1.3)$, где $\boldsymbol{h}(r, \rho)=$ $\left(h_{\ell j}(r, \rho)\right)_{\ell, j=1}^{s}-$ матрища-функция, элементы которой удовлетворяют условиям a) и b). Аналогичньй смысл имеет матричный оператор $\mathscr{H} \equiv \mathscr{H}_{s}$. Положим

$$
\widetilde{P}_{\tau_{1}, \tau_{2}}^{(s)}=\left(\widetilde{P}_{\tau_{1}, \tau_{2}} \delta_{\ell j}\right)_{\ell, j=1}^{s} \quad \text { и } \quad \widetilde{P}_{\tau}^{(s)}=\left(\widetilde{P}_{\tau} \delta_{\ell j}\right)_{\ell, j=1}^{s} .
$$

Символом оператора $\lambda I-H_{s}$ (и оператора $\lambda I-\mathscr{H}_{s}$ ) назовем матрицу-функцию

$$
\mathfrak{G}(\xi)=\lambda E-\left(\int_{0}^{\infty} h_{\ell j}(r, \rho) \rho^{-1 / p+i \xi} d \rho\right)_{\ell, j=1}^{s}, \quad \xi \in \mathbb{R}
$$

где $E$ - единичная матрища.

ПРЕДЛОЖЕНИЕ 3. Для того чтобы оператор $\lambda I-H_{s} \in \Pi\left\{\widetilde{P}_{\tau}^{(s)}\right\} \quad\left(\lambda I-\mathscr{H}_{s} \in\right.$ $\left.\Pi\left\{\widetilde{P}_{\tau_{1}, \tau_{2}}^{(s)}\right\}\right)$, необходимо и достаточно, чтобы выполнялись следующие условия:

1) $\operatorname{det} \mathfrak{G}(\xi) \neq 0$ для всех $\xi \in \dot{\mathbb{R}}$

2) все левые и правые индексы матрицы-функции $\mathfrak{G}(\xi)$ равны нулю.

ДокАЗАТЕЛьСТВо аналогично доказательству предложения 1.

\section{2. Многомерные интегральные операторы с однородными степени $(-n)$ я,драми}

В пространстве $L_{p}\left(\Omega_{n}\right)$ рассмотрим интегральньй оператор

$$
(K \varphi)(x)=\int_{\Omega_{n}} k(x, y) \varphi(y) d y, \quad x \in \Omega_{n},
$$

предполагая, что ядро $k(x, y)$ удовлетворяет следующим условиям:

$1^{\circ}$ однородности степени $(-n)$, т. е.

$$
k(\alpha x, \alpha y)=\alpha^{-n} k(x, y) \quad \forall \alpha>0 ;
$$

$2^{\circ}$ инвариантности относительно группы $S O(n)$ вращений пространства $\mathbb{R}^{n}$, т. е.

$$
k(\omega(x), \omega(y))=k(x, y) \quad \forall \omega \in S O(n) ;
$$

$3^{\circ}$ суммируемости, т. е.

$$
k=\int_{\mathbb{R}^{n}}\left|k\left(e_{1}, y\right)\right||y|^{-n / p} d y<\infty .
$$


Символом оператора $\lambda I-K$ назовем функцию $\sigma(m, \xi)$, заданную на компакте $\mathbb{Z}_{+} \dot{\times} \mathbb{R}$ равенством

$$
\sigma(m, \xi)=\lambda-\int_{\mathbb{R}^{n}} k\left(e_{1}, y\right) P_{m}\left(e_{1} \cdot y^{\prime}\right)|y|^{-n / p+i \xi} d y
$$

Далее, в пространстве $L_{p}\left(\Omega_{n}\right)$ определим проектор $P_{\tau}, 0<\tau<1$, по формуле

$$
\left(P_{\tau} \varphi\right)(x)= \begin{cases}\varphi(x), & \tau<|x|<1 \\ 0, & |x|<\tau\end{cases}
$$

Изучим вопрос о применимости к оператору $\lambda I-K$ проекционного метода по системе проекторов $\left(P_{\tau} ; P_{\tau}\right)$. Для этого в пространстве $L_{p}\left(\Omega_{n}\right)$ рассмотрим интегральное уравнение, порождаемое оператором $\lambda I-K$ :

$$
\lambda \varphi(x)=\int_{\Omega_{n}} k(x, y) \varphi(y) d y+f(x) .
$$

Поскольку функция $k(x, y)$ удовлетворяет условию $2^{\circ}$, то сушествует такая функция $k_{0}\left(r^{2}, \rho^{2}, t\right)$, что $k(x, y)=k_{0}\left(|x|^{2},|y|^{2}, x^{\prime} \cdot y^{\prime}\right)[6$, c. 36]. Учитьвая это и переходя в уравнении (2.3) к сферическим координатам $x=r \sigma, y=\rho \theta$, получим

$$
\lambda \Phi(r \sigma)=\int_{0}^{1} \int_{S_{n-1}} \frac{1}{r} D\left(\frac{\rho}{r}, \sigma \cdot \theta\right) \Phi(\rho \theta) d \rho d \theta+F(r \sigma),
$$

где

$$
\begin{gathered}
\Phi(r \sigma)=\varphi(r \sigma) r^{(n-1) / p}, \quad F(r \sigma)=f(r \sigma) r^{(n-1) / p} \\
D(\rho, t)=k_{0}\left(1, \rho^{2}, t\right) \rho^{(n-1) / p^{\prime}}
\end{gathered}
$$

Легко проверить, что

$$
\int_{0}^{\infty} \int_{-1}^{1}|D(\rho, t)| \rho^{-1 / p}\left(1-t^{2}\right)^{(n-3) / 2} d \rho d t<\infty .
$$

Умножая уравнение $(2.4)$ на $Y_{m \mu}(\sigma)$, интегрируя по единичной сффере и применяя формулу Функа-Гекке [6, с. 43], получим бесконечную диагональную систему одномернњх интегральных уравнений:

$$
\lambda \Phi_{m \mu}(r)=\int_{0}^{1} \frac{1}{r} D_{m}\left(\frac{\rho}{r}\right) \Phi_{m \mu}(\rho) d \rho+F_{m \mu}(r), \quad r \in(0,1),
$$

где $m \in \mathbb{Z}_{+}, \mu=1,2, \ldots, d_{n}(m)$,

$$
\begin{gathered}
\Phi_{m \mu}(r)=\int_{S_{n-1}} \Phi(r \sigma) Y_{m \mu}(\sigma) d \sigma, \quad F_{m \mu}(r)=\int_{S_{n-1}} F(r \sigma) Y_{m \mu}(\sigma) d \sigma \\
D_{m}(\rho)=\frac{2 \pi^{(n-1) / 2}}{\Gamma((n-1) / 2)} \int_{-1}^{1} D(\rho, t) P_{m}(t)\left(1-t^{2}\right)^{(n-3) / 2} d t .
\end{gathered}
$$


Рассмотрим в $L_{p}(0,1)$ оператор

$$
\left(K_{m} g\right)(r)=\int_{0}^{1} \frac{1}{r} D_{m}\left(\frac{\rho}{r}\right) g(\rho) d \rho, \quad r \in(0,1) .
$$

Легко проверить, что функция $D_{m}(\rho)$ удовлетворяет условию b) п. 1.2. Тогда оператор $K_{m}$ есть оператор вида (1.3).

Анализируя связь между уравнением (2.3) и системой (2.6), убеждаемся в справедливости следующих утверждений (см. также [5, с. 75-82]).

Лемма 1. 1) Oператор $\lambda I-K$ обратим в $L_{p}\left(\Omega_{n}\right)$ тогда и только тогда, когда оператор $\lambda I-K_{m}$ обратим в $L_{p}(0,1)$ для любого $m \in \mathbb{Z}_{+}$.

2) Oператор $P_{\tau}(\lambda I-K) P_{\tau}$ обратим в $P_{\tau}\left(L_{p}\left(\Omega_{n}\right)\right)$ тогда и только тогда, когда оператор $\widetilde{P}_{\tau}\left(\lambda I-K_{m}\right) \widetilde{P}_{\tau}$ обратим в $\widetilde{P}_{\tau}\left(L_{p}(0,1)\right)$ для любого $m \in \mathbb{Z}_{+}$.

ДокАЗАТЕльство. 1) Прежде всего покажем, что

$$
\lim _{m \rightarrow \infty}\left\|K_{m}\right\|=0
$$

В самом деле, норма оператора $K_{m}$ удовлетворяет неравенству (см. [5, с. 52])

$$
\left\|K_{m}\right\| \leqslant \int_{0}^{\infty}\left|D_{m}(\rho)\right| \rho^{-1 / p} d \rho .
$$

Из (2.7) и свойств многочленов Лежандра следует, что $D_{m}(\rho) \rightarrow 0$ для почти всех $\rho \in(0, \infty)$. Тогда, применяя мажорантную теорему Лебега, с учетом (2.5) получаем, что интеграл в правой части (2.9) стремится к нулю при $m \rightarrow \infty$. Следовательно, справедливо равенство (2.8).

Из (2.8) вытекает существование такого числа $M_{0} \in \mathbb{N}$, что для всех $m>M_{0}$ операторы $\lambda I-K_{m}$ обратимы.

Далее, в [5, с. 82] с учетом (2.8) доказьвается, что существует такое число $M \in \mathbb{N}$, что уравнение (2.3) в смысле разрешимости эквивалентно конечной системе одномерных уравнений $(2.6)$, где $m=0,1, \ldots, M ; \mu=1,2, \ldots, d_{n}(m)$. При этом число $M$ можно выбрать так, чтобы вьполнялось условие $M>M_{0}$. Так как система конечна, то оператор $\lambda I-K$ обратим тогда и только тогда, когда обратимы операторы $\lambda I-K_{m}$, $m=0,1, \ldots, M$. Все остальные операторы $\lambda I-K_{m}$ заведомо обратимы.

2) Вторая часть леммы доказывается аналогично.

ТЕорема 1. Для того чтобы оператор $\lambda I-K \in \Pi\left\{P_{\tau}\right\}$, необходимо и достаточно, чтобы оператор $\lambda I-K$ был обратим в $L_{p}\left(\Omega_{n}\right)$.

ДокАЗАТЕЛЬСтво. Необходимость следует из определения проекционного метода. Докажем достаточность. Пусть оператор $\lambda I-K$ обратим. Определим на $L_{p}\left(\Omega_{n}\right)$ проектор $\mathscr{P}_{M}$ равенством

$$
\left(\mathscr{P}_{M} \varphi\right)(x)=\sum_{m=0}^{M} \sum_{\mu=1}^{d_{n}(m)} \varphi_{m \mu}(|x|) Y_{m \mu}\left(x^{\prime}\right)
$$


и положим $\mathscr{Q}_{M}=I-\mathscr{P}_{M}$. Легко проверить, что проекторы $\mathscr{P}_{M}$ и $P_{\tau}$ коммутируют и подпространства $\operatorname{Im} \mathscr{P}_{M}$ и $\operatorname{Im} \mathscr{Q}_{M}$ инвариантны относительно оператора $\lambda I-K$. Положим

$$
\begin{array}{cl}
(\lambda I-K) \mathscr{P}=\left.(\lambda I-K)\right|_{\operatorname{Im} \mathscr{P}}, & (\lambda I-K)_{\mathscr{Q}}=\left.(\lambda I-K)\right|_{\operatorname{Im} \mathscr{Q}} \\
P_{\mathscr{P} \tau}=\left.P_{\tau}\right|_{\operatorname{Im} \mathscr{P}}, \quad P_{\mathscr{Q} \tau}=\left.P_{\tau}\right|_{\operatorname{Im} \mathscr{Q}} .
\end{array}
$$

В силу теоремы о поблочной применимости проекционного метода $[1$, с. 93$]$ для того чтобы оператор $\lambda I-K \in \Pi\left\{P_{\tau}\right\}$, необходимо и достаточно, чтобы

$$
(\lambda I-K) \mathscr{P} \in \Pi\left\{P_{\mathscr{P}}\right\} \quad \text { и } \quad(\lambda I-K)_{\mathscr{Q}} \in \Pi\left\{P_{\mathscr{Q} \tau}\right\} .
$$

Докажем, что $(\lambda I-K)_{\mathscr{Q}} \in \Pi\left\{P_{\mathscr{Q} \tau}\right\}$. Так как $I_{\mathscr{Q}} \in\left\{P_{\mathscr{Q} \tau}\right\}$, то найдется такое $\gamma>0$, что если $A \in \mathscr{L}\left(\operatorname{Im} \mathscr{Q}_{M}\right)$ и $\|A\|<\gamma$, то $\lambda I_{\mathscr{Q}}+A \in \Pi\left\{P_{\mathscr{Q}}\right\}[1$, с. 94]. Как известно [5, c. 81-82], число $M$ можно подобрать так, что норма $K_{\mathscr{Q}}$ будет меньше любого наперед заданного числа. Выберем число $M$ столь большим, чтобы вьполнялось неравенство $\left\|K_{\mathscr{Q}}\right\|<\gamma$, и зафиксируем $M$. Тогда $\lambda I_{\mathscr{Q}}-K_{\mathscr{Q}} \in \Pi\left\{P_{\mathscr{Q} \tau}\right\}$.

Покажем, что $(\lambda I-K) \mathscr{P} \in \Pi\left\{P_{\mathscr{P}}\right\}$. Так как оператор $\lambda I-K$ обратим в $L_{p}\left(\Omega_{n}\right)$, то по лемме 1 операторы $\lambda I-K_{m}$ обратимы в $L_{p}(0,1)$ для всех $m=0,1, \ldots, M$. Тогда в силу предложения 1 операторы $\lambda I-K_{m} \in \Pi\left\{\widetilde{P}_{\tau}\right\}$. Это значит, что для каждого $m$ найдется такое число $\tau_{m} \in(0,1)$, что при $0<\tau<\tau_{m}$ операторы $\widetilde{P}_{\tau}\left(\lambda I-K_{m}\right) \widetilde{P}_{\tau}$, действующие на $\widetilde{P}_{\tau}\left(L_{p}(0,1)\right)$, обратимы и

$$
\operatorname{sim}_{\tau \rightarrow 0}\left(\widetilde{P}_{\tau}\left(\lambda I-K_{m}\right) \widetilde{P}_{\tau}\right)^{-1} \widetilde{P}_{\tau}=\left(\lambda I-K_{m}\right)^{-1}
$$

Положим $\delta=\min _{0 \leqslant m \leqslant M}\left\{\tau_{m}\right\}$. Так как для всех $\tau \in(0, \delta)$ операторы

$$
\widetilde{P}_{\tau}\left(\lambda I-K_{m}\right) \widetilde{P}_{\tau}, \quad m=0,1, \ldots, M
$$

обратимы, то оператор $P_{\mathscr{P}}(\lambda I-K) \mathscr{P}_{\mathscr{P}_{\tau}}$, действующий на $P_{\mathscr{P} \tau}\left(\operatorname{Im} \mathscr{P}_{M}\right)$, обратим для всех $\tau \in(0, \delta)$. С помощью (2.10) легко проверяется, что

$$
s_{\tau \rightarrow 0} \lim _{\tau}\left(P_{\mathscr{P}}(\lambda I-K) \mathscr{P} P_{\mathscr{P}}\right)^{-1} P_{\mathscr{P}}=(\lambda I-K)_{\mathscr{P}}^{-1} .
$$

Таким образом, $(\lambda I-K) \mathscr{P} \in \Pi\left\{P_{\mathscr{P}}\right\}$. Теорема доказана.

В пространстве $L_{p}\left(\mathbb{R}^{n}\right)$ рассмотрим интегральньй оператор

$$
(\mathscr{K} \varphi)(x)=\int_{\mathbb{R}^{n}} k(x, y) \varphi(y) d y, \quad x \in \mathbb{R}^{n},
$$

ядро $k(x, y)$ которого удовлетворяет условиям $1^{\circ}-3^{\circ}$, и определим проектор $P_{\tau_{1}, \tau_{2}}, 0<$ $\tau_{1}<1,1<\tau_{2}<\infty$, по формуле

$$
\left(P_{\tau_{1}, \tau_{2}} \varphi\right)(x)= \begin{cases}\varphi(x), & \tau_{1}<|x|<\tau_{2}, \\ 0, & |x|<\tau_{1} \text { или }|x|>\tau_{2} .\end{cases}
$$


Символом оператора $\lambda I-\mathscr{K}$ назовем функцию $\sigma(m, \xi)$, заданную равенством (2.2). Известно [5, с. 78], что оператор $\lambda I-\mathscr{K}$ обратим в $L_{p}\left(\mathbb{R}^{n}\right)$ тогда и только тогда, когда $\sigma(m, \xi) \neq 0$ для всех $(m, \xi) \in \mathbb{Z}_{+} \dot{\times} \mathbb{R}$.

Далее, в пространстве $L_{p}(0, \infty)$ рассмотрим оператор

$$
\left(\mathscr{K}_{m} g\right)(r)=\int_{0}^{\infty} \frac{1}{r} D_{m}\left(\frac{\rho}{r}\right) g(\rho) d \rho, \quad r \in(0, \infty),
$$

где функция $D_{m}(\rho)$ определяется равенством (2.7). Ясно, что $\mathscr{K}_{m}$ есть оператор вида (1.2). Символом оператора $\lambda I-\mathscr{K}_{m}$ назовем функцию

$$
\sigma_{m}(\xi)=\lambda-\int_{0}^{\infty} D_{m}(\rho) \rho^{-1 / p+i \xi} d \rho, \quad \xi \in \mathbb{R} .
$$

Учитьвая формулу (2.7), получаем

$$
\sigma_{m}(\xi)=\lambda-\int_{\mathbb{R}^{n}} k\left(e_{1}, y\right) P_{m}\left(e_{1} \cdot y^{\prime}\right)|y|^{-n / p+i \xi} d y, \quad \xi \in \mathbb{R} .
$$

Таким образом, при фиксированном значении $m$ имеет место равенство

$$
\sigma_{m}(\xi)=\sigma(m, \xi) \quad \forall \xi \in \mathbb{R} .
$$

Следующее утверждение является аналогом леммы 1.

Лемма 2. 1) Oператор $\lambda I-\mathscr{K}$ обратим в $L_{p}\left(\mathbb{R}^{n}\right)$ тогда и только тогда, когда оператор $\lambda I-\mathscr{K}_{m}$ обратим в $L_{p}(0, \infty)$ для любого $m \in \mathbb{Z}_{+}$.

2) Onepamop $P_{\tau_{1}, \tau_{2}}(\lambda I-\mathscr{K}) P_{\tau_{1}, \tau_{2}}$ обратим в $P_{\tau_{1}, \tau_{2}}\left(L_{p}\left(\mathbb{R}^{n}\right)\right)$ тогда и только тогда, когда оператор $\widetilde{P}_{\tau_{1}, \tau_{2}}\left(\lambda I-\mathscr{K}_{m}\right) \widetilde{P}_{\tau_{1}, \tau_{2}}$ обратим в $\widetilde{P}_{\tau_{1}, \tau_{2}}\left(L_{p}(0, \infty)\right)$ для любого $m \in \mathbb{Z}_{+}$.

Теорема 2. Для того чтобъ оператор $\lambda I-\mathscr{K} \in \Pi\left\{P_{\tau_{1}, \tau_{2}}\right\}$, необходимо и достаточно, чтобы его символ $\sigma(m, \xi)$ удовлетворял следующим условиям:

1) $\sigma(m, \xi) \neq 0$ для всех $(m, \xi) \in \mathbb{Z}_{+} \dot{\times} \mathbb{R}$,

2) $\operatorname{ind}_{\xi} \sigma(m, \xi)=0$ для всех $m \in \mathbb{Z}_{+}$, где $\operatorname{ind}_{\xi} \sigma(m, \xi)$ - индекс функиии $\sigma(m, \xi)$ при фиксированном значении $m$.

ДокАЗАтЕЛЬСтво. Необходимость. Пусть $\lambda I-\mathscr{K} \in \Pi\left\{P_{\tau_{1}, \tau_{2}}\right\}$. Тогда оператор $\lambda I-\mathscr{K}$ обратим в $L_{p}\left(\mathbb{R}^{n}\right)$, а операторы $P_{\tau_{1}, \tau_{2}}(\lambda I-\mathscr{K}) P_{\tau_{1}, \tau_{2}}$ как операторы, действующие в $P_{\tau_{1}, \tau_{2}}\left(L_{p}\left(\mathbb{R}^{n}\right)\right)$, обратимы для достаточно малых $\tau_{1}$ и достаточно больших $\tau_{2}$, и

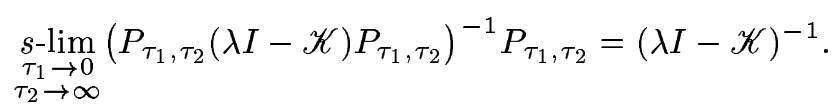

Тогда в силу леммы 2 оператор $\lambda I-\mathscr{K}_{m}$ обратим в $L_{p}(0, \infty)$, а оператор $\widetilde{P}_{\tau_{1}, \tau_{2}}(\lambda I-$ $\left.\mathscr{K}_{m}\right) \widetilde{P}_{\tau_{1}, \tau_{2}}$ обратим в $\widetilde{P}_{\tau_{1}, \tau_{2}}\left(L_{p}(0, \infty)\right)$ для всех $m \in \mathbb{Z}_{+}$. Кроме того, из условия $(2.12)$ легко следует, что

$$
\underset{\substack{s-\lim _{1 \rightarrow 0} \rightarrow \tau_{2} \rightarrow \infty}}{ }\left(\widetilde{P}_{\tau_{1}, \tau_{2}}\left(\lambda I-\mathscr{K}_{m}\right) \widetilde{P}_{\tau_{1}, \tau_{2}}\right)^{-1} P_{\tau_{1}, \tau_{2}}=\left(\lambda I-\mathscr{K}_{m}\right)^{-1} .
$$


Значит, $\lambda I-\mathscr{K}_{m} \in \Pi\left\{\widetilde{P}_{\tau_{1}, \tau_{2}}\right\}$ для всех $m \in \mathbb{Z}_{+}$. Учитьвая предложение 2 и равенство (2.11), убеждаемся в вьполнении условий 1), 2) данной теоремы.

Достаточность можно доказать так же, как в теореме 1. Мы, однако, дадим другое доказательство, основанное на самой теореме 1.

Определим на пространстве $L_{p}\left(\mathbb{R}^{n}\right)$ проектор $P$ по формуле

$$
(P \varphi)(x)= \begin{cases}\varphi(x), & |x| \leqslant 1 \\ 0, & |x|>1\end{cases}
$$

и положим $Q=I-P$. Представим оператор $\lambda I-\mathscr{K}$ в виде

$$
\lambda I-\mathscr{K}=P(\lambda I-\mathscr{K}) P+Q(\lambda I-\mathscr{K}) Q+T,
$$

где $T=-Q \mathscr{K} P-P \mathscr{K} Q-$ компактньй оператор [5, с. 380]. Рассмотрим оператор

$$
A=P(\lambda I-\mathscr{K}) P+Q(\lambda I-\mathscr{K}) Q
$$

и покажем, что $A \in \Pi\left\{P_{\tau_{1}, \tau_{2}}\right\}$. Положим

$$
A_{1}=\left.A\right|_{\operatorname{Im} P}, \quad A_{2}=\left.A\right|_{\operatorname{Im} Q}, \quad P_{\tau_{1}}=\left.P_{\tau_{1}, \tau_{2}}\right|_{\operatorname{Im} P}, \quad P_{\tau_{2}}=\left.P_{\tau_{1}, \tau_{2}}\right|_{\operatorname{Im} Q} .
$$

Заметим, что $A_{1}=\lambda I-K$, где $K$-оператор (2.1). Из условий 1$\left.), 2\right)$ следует, что опеpaтор $\lambda I-K$ обратим [5, с. 78]. Тогда по теореме $1 A_{1}=\lambda I-K \in \Pi\left\{P_{\tau_{1}}\right\}$. Аналогично доказьвается, что $A_{2} \in \Pi\left\{P_{\tau_{2}}\right\}$. В силу теоремы о поблочной применимости проекционного метода [1, с. 93] оператор $A \in \Pi\left\{P_{\tau_{1}, \tau_{2}}\right\}$. Далее, поскольку $A \in \Pi\left\{P_{\tau_{1}, \tau_{2}}\right\}$ и оператор $\lambda I-\mathscr{K}=A+T$ обратим, то по теореме о возмущении компактным оператором $[1$, с. 94$]$ оператор $\lambda I-\mathscr{K} \in \Pi\left\{P_{\tau_{1}, \tau_{2}}\right\}$. Теорема доказана.

ЗАмЕчАниЕ 2. Подчеркнем, что условие 1) является необходимым и достаточным условием для обратимости оператора $\lambda I-\mathscr{K}$, но для применимости проекционнного метода необходимо и достаточно вьполнения двух условий: 1) и 2).

Перейдем к изучению матричного случая. В $L_{p}^{s}\left(\Omega_{n}\right)$ рассмотрим оператор

$$
\left(K_{s} \varphi\right)(x)=\int_{\Omega_{n}} k(x, y) \varphi(y) d y, \quad x \in \Omega_{n},
$$

где $\boldsymbol{k}(x, y)=\left(k_{\ell j}(x, y)\right)_{\ell, j=1}^{s}-$ матрица-функция $s$-го порядка, элементы которой удовлетворяют условиям $1^{\circ}-3^{\circ}$. Аналогично в пространстве $L_{p}^{s}\left(\mathbb{R}^{n}\right)$ определяется оператор $\mathscr{K}_{s}$. Символом оператора $\lambda I-K_{s}\left(\lambda I-\mathscr{K}_{s}\right)$ назовем матрицу-функцию $\mathfrak{G}(m, \xi)$, заданную на компакте $\mathbb{Z}_{+} \dot{\times} \mathbb{R}$ равенством

$$
\mathfrak{G}(m, \xi)=\lambda E-\left(\int_{\mathbb{R}^{n}} k_{\ell j}\left(e_{1}, y\right) P_{m}\left(e_{1} \cdot y^{\prime}\right)|y|^{-n / p+i \xi} d y\right)_{\ell, j=1}^{s} .
$$

Определим проекторы $P_{\tau}^{(s)}$ и $P_{\tau_{1}, \tau_{2}}^{(s)}$ формулами

$$
P_{\tau}^{(s)}=\left(P_{\tau} \delta_{\ell j}\right)_{\ell, j=1}^{s}, \quad P_{\tau_{1}, \tau_{2}}^{(s)}=\left(P_{\tau_{1}, \tau_{2}} \delta_{\ell j}\right)_{\ell, j=1}^{s} .
$$


ТЕОрема 3. Для того чтобы оператор $\lambda I-K_{s} \in \Pi\left\{P_{\tau}^{(s)}\right\}$ (соответственно $\left.\lambda I-\mathscr{K}_{s} \in \Pi\left\{P_{\tau_{1}, \tau_{2}}^{(s)}\right\}\right)$, необходимо и достаточно, чтобы выполнялисв условия

1) $\operatorname{det} \mathfrak{G}(m, \xi) \neq 0$ для всех $(m, \xi) \in \mathbb{Z}_{+} \dot{\times} \mathbb{R}$

2) при любом фиксированном значении $m \in \mathbb{Z}_{+}$все левые и правые индексы матрицы-функиии $\mathfrak{G}(m, \xi)$ равны нулю.

ДокАЗАТЕЛЬСТво. Необходимость доказьвается так же, как и в теореме 2 с использованием предложения 3. Достаточность доказывается по той же схеме, что и в теоремe 1.

\section{СПИСОК ЦИТИРОВАННОЙ ЛИТЕРАТУРЫ}

[1] Гохберг И. Ц., Фельдман И. А. Уравнения в свертках и проекционные методы их решения. М.: Наука, 1971.

[2] Böttcher A., Silbermann B. Analysis of Toeplitz Operators. Berlin-Heidelberg-New York: Springer-Verlag, 1990.

[3] Prössdorf S., Silbermann B. Numerical Analysis for Integral and Related Operator Equations. Basel-Boston-Berlin: Birkhäuser, 1991.

[4] Авсянкин О. Г., Карапетянц Н. К. Многомерные интегральные операторы с однородньми степени $(-n)$ ядрами // Докл. РАН. 1999. Т. 368. №6. С. 727-729.

[5] Karapetiants N., Samko S. Equations with Involutive Operators. Boston-Basel-Berlin: Birkhäuser, 2001.

[6] Самко С. Г. Гиперсингулярные интегралы и их приложения. Ростов-на-Дону: Изд-во РГУ, 1984.

Ростовский государственный университет

Поступило

E-mail :

25.03.2002

avsyanki@ns .math.rsu.ru

nkarapet@ns .math.rsu .ru

Исправленный вариант

24.10 .2002 